\title{
Obesity and cancer
}

Donald C McMillan, Naveed Sattar, Mike Lean, Colin S McArdle

\section{Incidence and mortality}

Obesity is increasing at an alarming rate throughout North America and Europe and is associated with substantial morbidity and mortality. The condition has long been recognised as a risk factor for diabetes and cardiovascular disease, but not for developing cancer. A recent survey of the public by the American Cancer Society found that less than 5\% of respondents were aware of the increased cancer risk associated with overweight and obesity.

A recent meta-analysis showed that more than 70000 of the 3.5 million new cases of cancer each year in the European Union are attributable to overweight or obesity. This is likely to be a conservative estimate for two reasons. Firstly, only those tumours for which there was existing evidence to suggest a link between obesity and cancer (namely, for breast, colon, endometrium, prostate, kidney, and gallbladder) were included in the study. Secondly, the number of cancers attributable to obesity is likely to have increased because obesity levels have increased substantially since the publication of many of the studies included in this meta-analysis.

More recently, the American Cancer Society's prospective population based study of about 900000 subjects confirmed that obesity was directly associated with an increased risk of death from a variety of cancers.

In both men and women, obesity was significantly associated with higher death rates from cancer of the oesophagus, colon and rectum, gallbladder, pancreas, and kidney, independently of smoking. Obesity was also associated with an increased risk of death from cancers of the stomach and prostate in men and from cancers of the breast (postmenopausal), uterus, cervix, and ovary in women. The increased risk of cancer has been most clearly defined in the common cancers. For example, in males the risk of colorectal cancer increased up to $80 \%$ in those whose body mass index (weight in kilograms divided by height in metres squared) was greater than 30 .

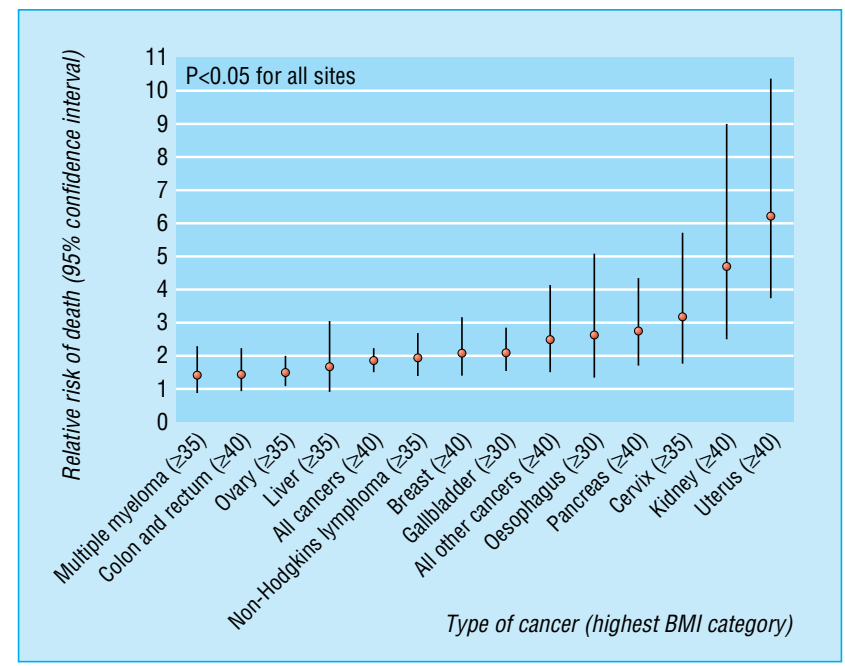

Mortality from cancer for US women, 1982-98, by body mass index (BMI) For each relative risk, the comparison was between women in the highest BMI category and women in the reference category (18.5 to 24.9). Adapted from Calle et al (see Further Reading box)
Percentage of cancer cases attributable to overweight and obesity in countries of the European Union, by cancer site. Adapted from Bergstrom et al (see Further Reading box)

\begin{tabular}{lcc} 
Site & Men & Women \\
\hline Breast & 0 & 8.5 \\
\hline Colon & 11.1 & 10.7 \\
\hline Endometrium & NA & 39.2 \\
\hline Prostate & 4.4 & NA \\
\hline Kidney & 25.5 & 24.5 \\
\hline Gall bladder & 24.8 & 23.7 \\
\hline All cancers & 3.4 & 6.4 \\
\hline
\end{tabular}

$\mathrm{NA}=$ not applicable.

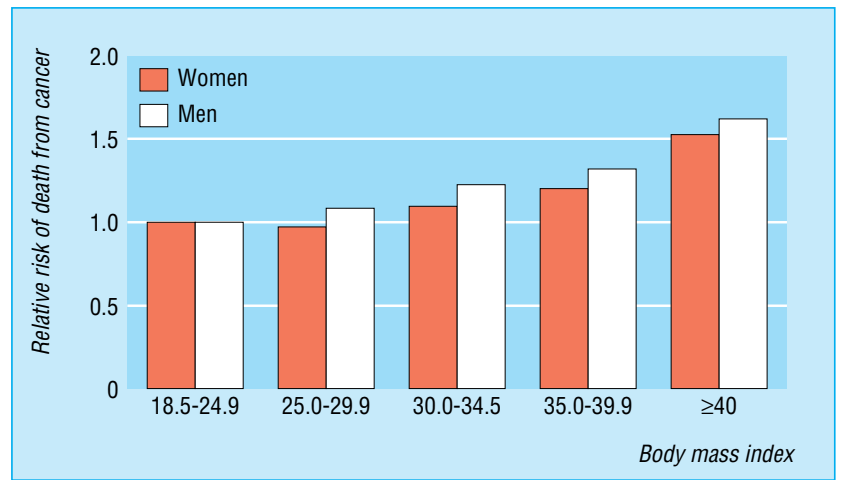

Contribution of overweight and obesity to mortality from cancer in the United States, 1982-98. Adapted from Adami H-O et al (N Engl J Med 2003;348:1623-4)

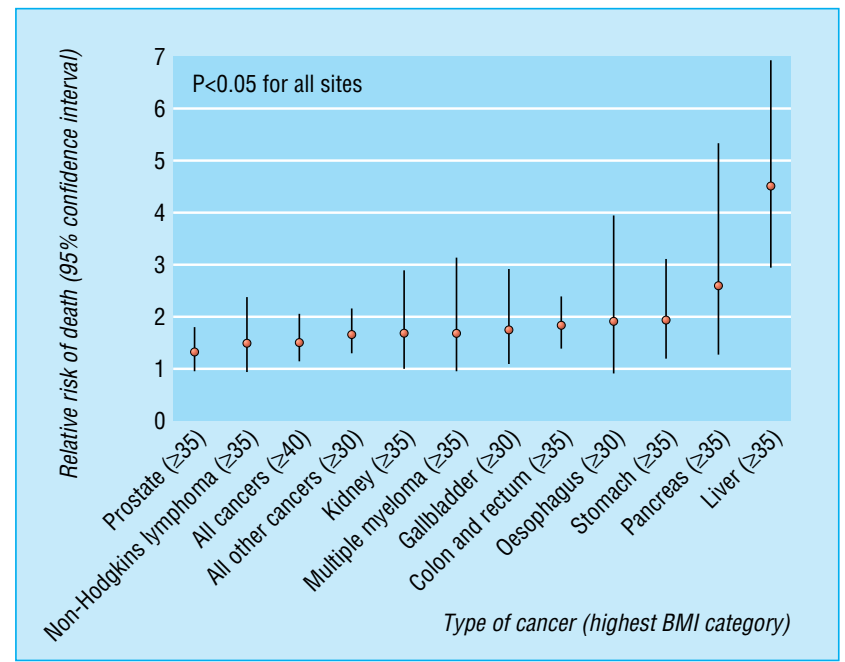

Mortality from cancer for US men, 1982-98, by body mass index (BMI). For each relative risk, the comparison was between men in the highest BMI category and men in the reference category (18.5 to 24.9). Adapted from Calle et al (see Further Reading box) 
On the basis of these results from the American Cancer Society, overweight and obesity in the United States is currently estimated to account for $14 \%$ of all deaths from cancer in men and $20 \%$ of those in women. Taken together, this means that 90000 cancer deaths could be prevented each year if Americans could maintain a healthy body weight. In Europe, 9000 of the 70000 cancer cases attributable to obesity and overweight ("avoidable" cases) are in the United Kingdom.

\section{Role of lifestyle factors}

\section{Diet}

Many studies have tried to document the health hazard that can be related to a diet high in calories and animal fat and low in fibre, fruit, and vegetables-a diet typical of Western market economies. Surveys of eating habits are consistently confounded by under-reporting, particularly among obese respondents.

Although initial studies suggested a strong association with total fat intake, particularly from animal sources, recent findings from retrospective and prospective epidemiological studies have been inconsistent. In addition, cereals with high fibre content have been associated with a lower risk of colorectal cancer, yet several large population based studies have failed to show benefit.

A more consistent association exists between reduced risk of cancer and increased consumption of a wide variety of fruit and vegetables; however, the relative quantities of each required to reduce risk is not known.

\section{Physical activity}

On the basis of existing evidence, public health organisations have issued guidelines for preventing chronic diseases including cancer. These recommendations include more exercise during daily routine-for example, use stairs, walk to work, walk in the park, and do more physical activity around the home (such as gardening). Specifically, individuals are advised to do some sustained physical activity-such as brisk walking for 30-40 minutes-at least five days a week. For people with known cardiovascular disease, a lower level of physical activity may be of benefit- however, they should do this only after consultation with their doctor.

A recent population based study of adults in 48 states in North America has shown an increase in obesity in all states and a decrease in physical activity in only 11 . This suggests that obesity is now primarily diet induced, the result of a sustained excess of energy rich foods with high fat and refined carbohydrate content, as well as an insufficient consumption of fruit and vegetables. This is compounded by increasingly sedentary lifestyles; paradoxically, appetite is increased in inactive people.

In Europe, the position is less clear as some evidence exists to suggest that the lack of physical activity may make a more important contribution to the development of obesity.

\section{Mechanisms}

Several plausible biological mechanisms have been suggested to explain the relation between obesity and the increased risk of developing cancer, including the increased concentrations of endogenous hormones. These hormones (sex steroids, insulin, and insulin growth factor-1) are increased with the accumulation of body fat and are important in the control of growth, differentiation, and metabolism of cells. In particular, hyperinsulinaemia would link obesity related cancer with the high energy, highly processed, carbohydrate and animal fat diet
Given that obesity rates in European countries are fast catching up with those in North America, similar numbers of patients will probably be exposed to an increased risk of developing cancer

The relation between obesity and the incidence of cancers is likely to be linked to aspects of lifestyle-in particular, diet and physical activity

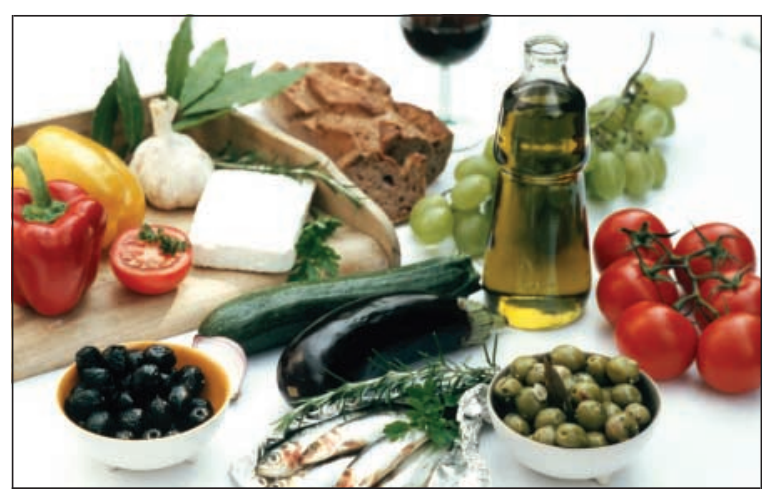

Southern European diets-characterised by a low intake of animal fat and a high intake of fish, olive oil, vegetables, fruits, and grain-are consistently associated with lower rates of cancer

Almost 170 observational studies have evaluated the relation between physical activity and the risk of developing cancer. The results of these studies provide convincing evidence that increased physical activity is associated with a reduction in several common solid tumours, including colon cancer

The balance between energy intake and expenditure

- In some people the major issue is overeating for the physical activity they perform

- In others, the major issue is too little physical activity and a failure to reduce their food intake accordingly

- Therefore, in both cases, obesity seems to result from an inability to match energy intake and energy expenditure

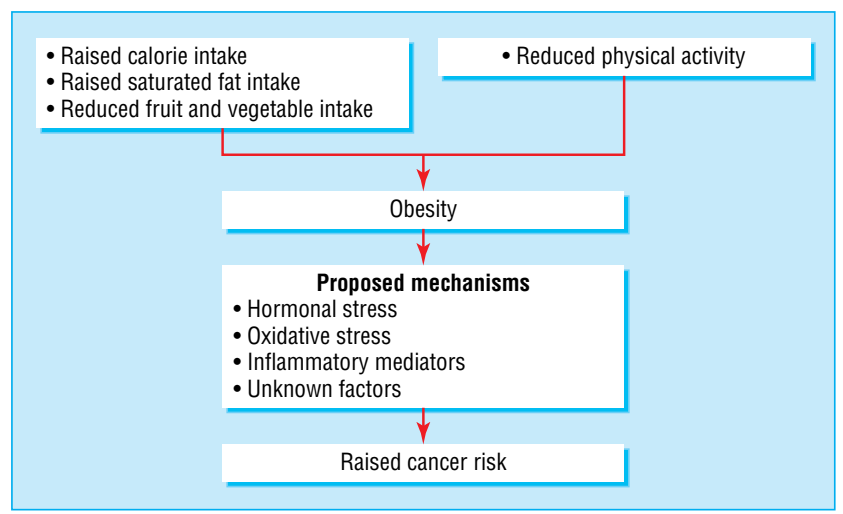

Links between obesity, lifestyle factors, and risk for cancer 
typical of Western society. It is therefore of interest that patients with type 2 diabetes are known to have increased rates of cancer.

Chronic inflammation is recognised to be an important factor in the initiation and promotion of cancer cells. Recent studies suggest that adipocytes are a potent source of pro-inflammatory cytokines and that obesity is a key component explaining the presence of a chronic, low grade, systemic inflammatory response in the general population.

The presence of a systemic inflammatory response (as evidenced by an increase in circulating $\mathrm{C}$ reactive protein concentrations) has been shown, for example, to be associated with an increased risk of colorectal cancer. These observations are consistent with the results of recent randomised trials showing that the use of conventional non-steroidal anti-inflammatory drugs reduce the incidence of polyp formation. Therefore, the presence of a chronic systemic inflammatory response provides a potential direct link between obesity and increased development of cancer. Obesity is linked to enhanced oxidative stress, in part through the inflammatory process, and increased oxidative damage to DNA could raise cancer risk.

\section{Guidelines}

Sufficient evidence exists that the incidence of cancer can be reduced by changes in lifestyle to include a healthy diet and physical activity. The International Union Against Cancer (UICC) has issued dietary guidelines for reducing cancer risk.

\section{Conclusion}

Obesity in the United Kingdom is increasing rapidly and will lead to an increase not only in the incidence of diabetes and cardiovascular disease but also in the incidence of cancer. It remains to be seen whether a documented excess risk of death due to cancer among overweight and obese people will provide additional motivation for controlling body weight around the world.

The photograph is published with permission from Alix/Phanie/Rex.

Donald C McMillan is senior lecturer and Colin S McArdle is an honorary professor at the university department of surgery at the Royal Infirmary, Glasgow.

The ABC of Obesity is edited by Naveed Sattar

(nsattar@clinmed.gla.ac.uk),professor of metabolic medicine, and Mike Lean, professor of nutrition, University of Glasgow. The series will be published as a book by Blackwell Publishing in early 2007 .
Guidelines of the International Union Against Cancer

Consume a lifelong, varied diet rich in plant foods

- Include fruits and vegetables in every meal

- Eat fruits and vegetables as snacks

- Substitute beans for meat

- Select wholegrain products rather than refined grains

- Include high fibre foods in the diet

Restrict intake of fatty foods

- Meat portions should be small relative to servings of plant food

- Meat should be trimmed of fat

- Eat fish and poultry rather than meats high in saturated fats

- Limit intake of fried food

- Limit additions of fats and oils to prepared foods

- Limit or avoid alcohol consumption

Store and prepare foods in ways that reduce contamination

- Fresh foods should be properly cleaned before consumption

- Perishable foods should be refrigerated

- Limit consumption of salted, nitrite treated, smoked, and pickled foods

- Avoid charred foods, which can be carcinogenic

- Add less salt during food preparation and eating

Balance dietary intake and energy expenditure to avoid excesses of high or low weight

- Eat small portions of high energy foods

- Exercise to maintain weight

Do not rely on supplementary vitamins and minerals as substitutes for balanced and adequate diet

- Maximise intake of essential nutrients by inclusion of vitamin and mineral rich foods

- Use supplements only for needs not adequately met by diet

\section{Further reading and resources}

- Bergstrom A, Pisani P, Tenet V, Wolk A, Adami HO. Overweight as an avoidable cause of cancer in Europe. Int J Cancer 2001;91:421-30.

- Calle EE, Rodriguez C, Walker-Thurmond K, Thun MJ. Overweight, obesity, and mortality from cancer in a prospectively studied cohort of US adults. N Engl J Med 2003;348:1625-38.

- Vigneri P, Frasca F, Sciacca L, Frittitta L, Vigneri R. Obesity and cancer. Nutr Metab Cardiovasc Dis 2006;16:1-7.

- www.iotf.org (International Association for the Study of Obesity)

- www.uicc.org (International Union Against Cancer)

- www.wcrf.org (World Cancer Research Fund)

- www.iarc.fr (International Agency for Research on Cancer)

Competing interests: For series editors' competing interests, see the first article in this series.

BMJ 2006;333:1109-11

\section{Complaints, Shetland Islands style}

The gravestone inscription below confirms that naming and shaming has been part of the Shetland Islands complaints procedure for some years now:

Donald Robertson (1785-1848)

His death was much regretted which was caused by the stupidity of Laurence Tulloch in Clothister (Sullom) who sold him nitre instead of Epsom salts by which he was killed in the space of 5 hours after taking a dose of it.

Paul Brooksby consultant cardiologist, Mid Yorkshire Hospitals NHS Trust, Pontefract General Infirmary, Pontefract (paul.brooksby@btinternet.com)
We welcome articles up to 600 words on topics such as A memorable patient, A paper that changed my practice, My most unfortunate mistake, or any other piece conveying instruction, pathos, or humour. Please submit the article on http://submit.bmj.com Permission is needed from the patient or a relative if an identifiable patient is referred to. We also welcome contributions for "Endpieces," consisting of quotations of up to 80 words (but most are considerably shorter) from any source, ancient or modern, which have appealed to the reader. 